Inhalt

Onkologie 2012;35(suppl 5):1

\title{
2 Vorwort
}

Bernhard Wörmann, Berlin

3 Umsetzung integrativer Konzepte - eine Bestandsaufnahme

3 Integrative Onkologie im Kontext eines universitären Cancer Centers Verantwortlicher Autor: Matthias Rostock, Hamburg

6 Integrative Onkologie im Kontext einer Praxis Verantwortlicher Autor: Friedrich Overkamp, Recklinghausen

9 Die Bedürfnisse der Patienten Verantwortlicher Autor: Markus Horneber, Nürnberg

12 Was nehmen wir nicht (ausreichend) wahr?

12 Sport und Bewegung im Therapiekonzept Verantwortlicher Autor: Freerk T. Baumann, Köln

14 Aspekte der Ernährung bei Krebserkrankungen Verantwortlicher Autor: Jann Arends, Freiburg i.Br.

16 Gleichklang von Körper und Seele: Die Mind-Body-Medizin Verantwortliche Autorin: Anna Paul, Essen

17 Der Beitrag der traditionellen asiatischen Medizin zur Integrativen Onkologie

Verantwortlicher Autor: Hans Lampe, Rostock

21 Modellprojekte der Integrativen Onkologie

21 Das Kompetenznetz «Komplementärmedizin in der Onkologie - KOKON»

Verantwortlicher Autor: Martin Wilhelm, Nürnberg

22 Das «Essener Modell»

Verantwortlicher Autor: Gustav Dobos, Essen

26 Der Blick nach vorn ...

26 Brauchen wir eine neue ärztliche Ethik?

Verantwortlicher Autor: Herbert W. Kappauf, Starnberg

27 Qualitätsstandards in der Integrativen Onkologie Verantwortliche Autorin: Jutta Hübner, Frankfurt/M.

II Impressum

U3 Autorenverzeichnis 